\title{
Evaluación Clínica y Radiográfica de distintas Pastas de Obturación Radicular en Dientes Primarios
}

Clinical and radiographic evaluation of different pasta Radicular Shutter in Primary Teeth

Fecha de Recepción 25 de noviembre de 201 I
Avaliação clínica e radiográfica de massas diferente Obturação Radicular em dentes Primários
Gabriela Quintero de Lucas

Profesora Titular Cátedra de Odontopediatría. Facultad de Odontología Universidad

Nacional del Nordeste. FOUNNE.

María Susana Discacciatti de Lértora

Profesora Adjunta Cátedra de Odontopediatría. FOUNNE.

Miguel Escobar Auxiliar Docente Cátedra de Odontopediatría FOUNNE.

María Lorena Cardoso Auxiliar Docente Cátedra de Odontopediatría FOUNNE.
Aceptado para su publicación 21 de junio de 2012

\section{Resumen}

El objetivo de este estudio longitudinal fue evaluar clínica y radiograficamente el éxito, a los 6 y 12 meses, de diferentes pastas de relleno in molares primarios sometidos a pulpectomias. Cincuenta y cuatro molares primarios de 47 niños (edad promedio $5.8 \pm 1.2$ años) fueron distribuidos aleatoriamente a los siguientes grupos: OZinc Eugenol (OZE), iodoformo/glicerina (IG), Vitapex, Pasta de Guedes Pinto. El tratamiento se considero exitoso ante la presencia de un diente asintomático y radiográficamente se observo una disminución o ausencia de cambios en el defecto radiolucido patológico preexistente. El éxito total fue del $91 \%$, no demostrándose diferencias significativas entre el éxito clínico ( $p \leq 0.2069$ vs. $p \leq 0.1110$ ) y radiográfico ( $p \leq 0.3$ II 3 vs. $p \leq 0.3220)$ a los 6 y 12 meses. A los 12 meses el Vitapex mantuvo el $100 \%$ de las piezas, seguidos por la pasta de iodoformo/glicerina con 93\%, Pasta de Guedes Pinto con $86 \%$ y el OZE con $83 \%$.

Conclusión: $\mathrm{Si}$ bien todas las pastas de relleno demostraron ser efectivas, aquellas conteniendo materiales iodoformados fueron ligeramente superiores a la Pasta de Guedes y el OZE al permitir la permanencia de los molares primarios asintomáticos tanto clínica como radiográficamente a los 12 meses.

\section{Palabras claves}

Necrosis pulpar, pulpectomías, dentición primaria, pastas endodontica. 


\section{Summary}

The purpose of this longitudinal study was to evaluate, clinical and radiographic success rate of different filling pastes in pulpectomized primary molars at 6 and 12 months. Fifty four primary molars from 47 children (average age $5.8 \pm 1.2$ years) were allocated to the following groups: Zinc Oxide/Eugenol (0ZE), lodoform and glycerine, Vitapex and Guedes Pinto's Paste. Treatment was considered success if clinically the tooth was asymptomatic and radiographs showed decrease or no change in pre-existing pathologic radiolucent defect. The overall success rates were $91 \%$ at 12 months.

The obtained results demonstrated a total success of $91 \%$. In the total groups there was no statistically significant differences in clinical ( $p \leq 0.2069$ vs. $p \leq 0.1110)$ and radiographic ( $P$ $\leq 0.3113$ vs. $p \leq 0.3220$ ) success at 6 and 12 months. At 12 months Vitapex success rate was $100 \%$, lodoform and glycerin $93 \%$, Guedes Paste $86 \%$ and OZE $83 \%$.

Conclusion: All pastes proved effective, those containing iodoform were slightly higher than the Pasta de Guedes and OZE allowing retention of primary molars clinically asymptomatic and radiographically at 12 months.

\section{Key words}

Pulpectomy, primary dentition, necrotic tissue, endodontic paste.

\section{Resumo}

O objetivo deste estudo longitudinal foi avaliar - sucesso clínico e radiográfico em 6 e 12 meses, massas de preenchimento diferente em molares decíduos submetidos a pulpectomia. Cinqüenta e quatro molares decíduos de 47 crianças (idade média de 5,8 \pm I,2 anos) foram randomizados para os seguintes grupos: Eugenol OZinc (Oze), iodofórmio/glicerol (IG), Vitapex, Pasta Guedes Pinto. $O$ tratamento foi considerado bem sucedido na presença de um dente assintomático e radiograficamente observada uma diminuição ou nenhuma alteração no defeito patológicas existentes radiolúcida. A taxa de sucesso foi de $91 \%$, demonstrando não haver diferença significativa entre o sucesso clínico ( $p \leq 0,2069$ vs $P \leq 0,1$ I I 0$)$ e radiográfica $(P \leq 0,3 \mid 13$ vs $P \leq 0,3220)$ aos 6 e
I 2 meses. Aos I 2 meses o Vitapex mantido 100\% das peças, seguido pelo iodofórmio colar/93\% de glicerina, Guedes Pinto colar com $86 \%$ e $83 \%$ com Oze.

Conclusão: Enquanto a massa preenchendo todos demonstrou ser eficaz, os materiais que contenham iodo foram ligeiramente maiores do que o Guedes e cole Oze para permitir a retenção de molares decíduos assintomáticos clinicamente e radiograficamente em 12 meses.

\section{Palaviras chaves}

Necrose pulpar, pulpectomia, dentes decíduos, pastas endodônticas.

\section{Introducción}

La perdida de piezas dentarias en la dentición temporaria está asociada principalmente a la presencia de caries con compromiso pulpar. La caries, en un diente primario, es un proceso progresivo y rápido que, debido al reducido grosor del esmalte y dentina, afecta rápidamente al tejido pulpar produciendo una respuesta inflamatoria, inicialmente de tipo reversible, y posteriormente sino se trata, conduce a alteraciones patológicas irreversibles que afectan en los molares, primero a la parte inter radicular del periostio y después a la porción radicular de la pulpa.

Las pulpectomías o terapia endodóntica están indicadas en dientes temporarios uni o multiradiculares con evidencia clínica y radiográfica de la presencia de tejido pulpar cameral y/o radicular necrosado o infectado crónicamente $y$ en piezas con exposición pulpar en las que, realizada la amputación de la pulpa cameral, la porción radicular presenta signos de hiperemia o evidencia de necrosis. Su realización está contraindicada en aquellos casos de reabsorción radicular interna o externa avanzada e infección que afecte la cripta del diente sucedáneo, como así también en pacientes con enfermedades sistémicas o inmunosuprimidos.

La realización de las pulpectomías o tratamiento del/los conductos radiculares en molares primarios constituyen un reto, debido a sus características anatómicas. Poseen raíces delgadas y frecuentemente curvadas con gran cantidad de conductos accesorios, a nivel de la furca, que parten del suelo de la cavidad pulpar ${ }^{2}$. Ringelstein y Paras $^{3,4}$ encuentran una prevalencia de $42.7 \%$, 
ubicándose el $20 \%$ en la superficie interna y el $50 \%$ en la superficie externa. La presencia de estos conductos accesorios, en el área de la furcación, son clínicamente importantes ya que proveen una comunicación con el tejido periodontal permitiendo la afectación del hueso interradicular antes que el tejido periapical, además de contribuir a la penetración de los medicamentos colocados en la pulpa cameral, hacia el hueso ${ }^{5}$.

Las condiciones existentes en el conducto radicular, después de una necrosis del tejido pulpar, favorecen el crecimiento bacteriano compuesto mayormente por anaerobios obligados Gram negativos $y$, en menor proporción, por aerobios. Estos microorganismos producen sustancias toxicas como son los factores de virulencia y endotoxinas (lipopolisacáridos) liberadas a los tejidos apicales y periapicales, que estimulan la reabsorción ósea ${ }^{6}$. Los procesos osteolíticos persistentes en la región de la furca, en los molares primarios, o en el ápice en las piezas anteriores pueden llevar, en caso de progresión, a la destrucción del lecho óseo del germen permanente, con el consecuente peligro de afectar el desarrollo del germen mismo.

Algunos investigadores sostienen que la instrumentación biomecánica, por si sola, no es capaz de producir la eliminación completa de los microorganismos del complejo sistema radicular, siendo importante la utilización la medicamentos con propiedades antibacterianas que ayuden a eliminar la infección remanente en los espacios radiculares y en las paredes de los conductos, como así también en áreas inaccesibles, jugando un importante rol las pastas de obturación o de relleno del conducto, en el pronóstico del tratamiento endodóntico en un diente primario infectado ${ }^{7}$.

El éxito de estos tratamientos se basa en un estricto criterio de selección de la pieza, adecuada instrumentación biomecánicas y obturación con un cemento adecuado, estableciéndose como criterio de éxito la ausencia de sintomatología tanto clínica como radiográfica permaneciendo la pieza tratada hasta su normal recambio.

El carácter provisional del diente primario supone que el material de obturación de los conductos debe cumplir exigencias especiales. Diversos autores $^{8,9}$ han propuesto las propiedades que deberían tener estas pastas para su utilización en dientes primarios:
- reabsorberse a un ritmo similar a la pieza dentaria tratada,

- ser inocua para los tejidos periapicales y para el germen del diente permanente en desarrollo,

- reabsorberse rápidamente en caso de sobrepasar el ápice dentario

- ser altamente antiséptica.

- Ser de fácil manipulación que permita rellenar fácilmente todos los conductos presentes,

- adherirse a las paredes del conducto,

- no sufrir fenómenos de contracción,

- ser radiopaco

- evitar el cambio de color de la pieza dentaria afectada.

- no producir una masa compacta que interfiera con la erupción del diente permanente al endurecer.

Si bien, en la actualidad no se dispone de un material que reuna todas estas propiedades, numerosas pastas han sido utilizadas, cada una con características propias, siendo los más utilizados aquellos cuyos componentes principales son Óxido de Zinc - Eugenol, Yodoformo e Hidróxido de Calcio.

El objetivo de este trabajo fue verificar la respuesta biológica, de distintas pastas de obturación, en el tratamiento de las pulpitis irreversibles o necrosis pulpar en molares primarios.

\section{Materiales y Métodos}

\section{Muestra}

Este estudio fue realizado en 47 niños, con una edad promedio de 5.8 años de edad, que concurrieron a la Clínica de la Cátedra de Odontopediatría de la Facultad de Odontología de la U.N.N.E y a la Clínica de Bebés, Niños y Adolescentes de la Fundación Escuela Posgrado - Universidad Católica de Salta en la ciudad de Resistencia.

\section{Selección de pacientes}

Previa autorización y consentimiento informado por parte de los padres y/o tutores, a todos los niños se les realizó un examen inicial (evaluación pre-experimental) el que consistió en la toma de una minuciosa historia médica y dental, seguido de examen clínico y toma de radiografías periapicales. Aquellos pacientes que reunían las pautas establecidas en los criterios de inclusión fueron informados, conjuntamente con 
los padres de los procedimientos a realizar y los objetivos del presente trabajo.

\section{Criterios de Inclusión}

Niños sanos, colaboradores y con buena conducta, con edades comprendidas entre los 4 a 8.5 años con piezas dentarias con pulpas irreversiblemente inflamadas.

Piezas dentarias con sintomatología clínica como dolor espontáneo, presencia de fístula, edema, movilidad, dolor a la presión.

Piezas que al realizarse la amputación de pulpa coronaria presentan un sangramiento profuso indicando la presencia de pulpa inflamada radicularmente, o bien, que al realizarse la apertura no presenten tejido pulpar remanente o presenten pus en los conductos.

Piezas dentarias con presencia radiográfica de: reabsorción radicular fisiológica avanzada, signos de necrosis pulpar con o sin presencia de zonas radiolúcidas en la furcación, engrosamiento del ligamento periodontal y/o reabsorción radicular interna, o cualquier otra evidencia de lesión patológica radicular con o sin caries.

Pieza dentaria afectada que pueda ser restauradas mediante la colocación de coronas de acero inoxidable.

\section{Criterios de exclusión}

Pieza no restaurable con proceso patológico que se extienda cercano al germen dentario permanente, con evidencia de extensa reabsorción radicular externa $o$ interna.

\section{Diseño experimental}

Este estudio se realizó en 54 molares primarios, divididos aleatoriamente en los siguientes grupos:

Grupo I: Obturados con oxido de zinc eugenol Grupo 2: Obturados con yodoformo y glicerina. Grupo 3: Obturados con hidróxido de calcio y yodoformo (Ultrapex®).

Grupo 4: Oturados con Pasta de Guedes (yodoformo, paramonoclorofenol y Rifocina).

Se realizaron controles, a los 6 y 12 meses, tanto clínicos como radiográficos, por un único operador. El éxito fue considerado cuando se cumplieron los siguientes parámetros:

Criterio clínico: ausencia de dolor, de sensibilidad a la presión y tejidos blandos sanos sin edema o inflamación, tracto fistulosos o enrojecimiento.
Criterio radiográfico:presencia de resolución de radio lucidez patológica preoperatoria interadicular o periapical por la aparición de áreas de calcificación u osificación, ausencia de nuevas áreas patológicas radiolúcidas, detención de la reabsorción radicular externa patológica, reabsorción de la pasta en caso de haberse extruido fuera del ápice radicular.

\section{Técnica para la realización de las pulpectomías}

Se realizó de acuerdo a la técnica descripta por O'Riordan y Coll (10), siguiéndose los siguientes pasos:

- Toma de radiografía periapical.

- Anestesia y aislamiento de la pieza a tratar.

- Eliminación del tejido cariado mediante fresa redonda grande $\mathrm{N}^{\circ} 6$ u 8.

- Eliminación del techo cameral con una fresa 330 de carburo tungsteno colocada en la turbina.

- Eliminación de la pulpa cameral con cucharillas afiladas.

- Lavado y secado de la cavidad cameral con solución salina normal y torundas de algodón estériles.

- Regularización de las paredes camerales mediante una fresa $N^{\circ} 170 \circ 169 \mathrm{~L}$ para turbina, quedando las paredes lisas y sin retenciones con una divergencia hacia oclusal.

- Medición de la longitud radicular y colocación de los topes tanto en las limas como en los escariadores.

- Eliminación del tejido radicular con escariadores dentados.

- Alisado de las paredes, con limas de Hedstrom, iniciándose con la de tamaño I5, mediante movimientos hacia fuera y adentro, no excediéndose el tamaño de 35. Los conductos palatinos de los molares superiores se instrumentaron hasta la lima 50 , manteniéndose $1 \mathrm{~mm}$. por debajo del ápice.

- Lavado e irrigación de los conductos: Después de cada cambio de lima se irrigaron los conductos con $2 \mathrm{ml}$. de solución salina normal con una aguja de diámetro $2 \mathrm{I}$ en una jeringa de $10 \mathrm{ml}$. Completada la instrumentación se realizó una irrigación final con $10 \mathrm{~mL}$ de solución salina.

- Secado de los conductos con puntas de papel. 
- Obturación de los conductos: Se realizó manualmente, colocando la pasta en la última lima utilizada y haciendo presión con torundas de algodón, hasta asegurar haber llenado el conducto radicular, a excepción de la colocación del Vitapex que fue introducido con el dispositivo provisto.

- Obturación de la cámara pulpar y cementado de corona de acero inoxidable: La cámara pulpar se rellenó con pasta de oxido de zinc eugenol reforzado, obturándose la pieza dentaria con una corona de acero inoxidable cementada con ionómero vítreo.

- Tomaderadiografíaperiapicalpostoperatoria.

Análisis Estadístico: Se utilizó la prueba de Chicuadrado a fin de establecer diferencias significativas entre el éxito clínico y radiográfico a los 6 y
12 meses demostrado por cada una de las pastas utilizadas.

\section{Resultados}

Este estudio fue realizado en 47 niños de los cuales 27 eran de sexo femenino y 20 masculino, con un promedio de $5.8 \pm 1.2$ años, finalizando a los 12 meses 45 niños. Se trataron 54 molares primarios, de los cuales 42 eran segundos molares primarios y 12 primeros molares, detallándose en la Figura I las piezas tratadas.

La Tabla I representa el total de piezas, a los 6 y 12 meses en cada uno de los grupos. Del total de piezas tratadas, a los 6 meses, 2 primeros molares inferiores debieron ser extraídos, pertenecientes a los grupos I y 2, por presentar sintomatología tanto clínica como radiográfica. A los 12 meses se

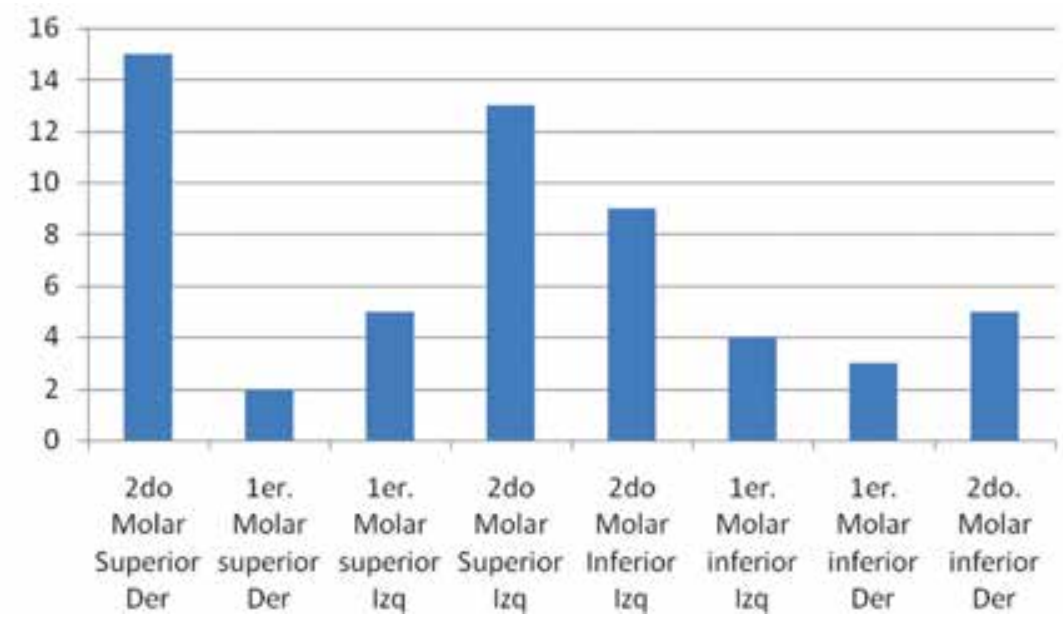

Figura I: Discriminación de los molares en el estudio

Tabla I

\begin{tabular}{|c|c|c|c|}
\hline Grupo & Inicial & $\mathbf{6}$ meses & 12 meses \\
\hline Grupo I & 12 & $11(92 \%)$ & $10(83 \%)$ \\
\hline Grupo 2 & 15 & $14(93 \%)$ & $14(93 \%)$ \\
\hline Grupo 3 & 13 & $12(92 \%)$ & $13(100 \%)$ \\
\hline Grupo 4 & 14 & $13(93 \%)$ & $12(86 \%)$ \\
\hline Totales & 54 & $50(93 \%)$ & $49(91 \%)$ \\
\hline
\end{tabular}

Tabla || |.

\begin{tabular}{|c|c|c|}
\hline & 6 meses & 12 meses \\
\hline Examen Clínico & $P \leq 0.2069$ & $P \leq 0.1110$ \\
\hline Examen Radiográfico & $P \leq 0.3113$ & $P \leq 0.3220$ \\
\hline
\end{tabular}


extrajeron I segundo molar superior, obturado con OZE y 2 molares, I primer molar superior y otro inferior, obturados con Pasta de Guedes. La diferencia observada en la cantidad de piezas en los controles de los 6 y 12 meses en el grupo 3 y 4 se debió a la no concurrencia, por enfermedad, de uno de los pacientes con 2 piezas obturadas. Los fracasos clínicos estuvieron asociados a presencia de movilidad dentaria y persistencia de un tracto fistuloso. En los grupos obturados con Oxido de Zinc Eugenol y lodoformo/glicerina estos se observaron tanto a los 6 como a los 12 meses, en tanto en el grupo obturado con Pasta de Guedes se observaron a los 12 meses.

Radiográficamente las piezas dentarias extraídas presentaban gran perdida ósea tanto interradicular como externa, coincidente con la movilidad observada clínicamente.

La Tabla 2 corresponde a los valores obtenidos, mediante el Chi Cuadrado de Pearson, a los 6 y 12 meses tanto clínica como radiográficamente, no demostrándose diferencias significativas entre estos parámetros en cada una de las pastas evaluadas.

\section{Discusión}

El objetivo primario de la pulpectomías, en dientes primarios, es la eliminación de los microorganismos interradiculares a fin de mantener la pieza dentaria primaria y sus tejidos de soporte en función e integridad hasta su normal recambio. El éxito de estos tratamientos, en dientes con pulpitis irreversible o con necrosis pulpar, depende de la eliminación de los microorganismos en todo el conducto radicular, procedimiento difícil de lograr por las características anatómicas y morfológicas complejas que presentan los molares primarios, a nivel radicular, que impiden la completa eliminación de las bacterias y sus productos localizados en los túbulos dentinarios ${ }^{12}$ lo que hace que la medicación utilizada debe poseer características tales como ser antiséptica a largo plazo, no tóxica y con capacidad de crear condiciones favorables para la reparación tisular además, de no alterar el desarrollo del germen permanente ${ }^{13}$.

En estos tratamientos los controles mediatos juegan un rol importante para evaluar la evolución del éxito o fracaso de los mismos. La Academia Americana de Odontopediatría establece que, en las piezas con pulpitis irreversibles o necrosis pulpar, los signos y síntomas clínicos deben resolverse a las pocas semanas post tratamiento, en tanto que la imagen radiográfica de un proceso infeccioso debe resolverse en 6 meses, evidenciado por la deposición de hueso en el área radiolúcida pretratamiento, debiendo realizarse la extracción en presencia de una marcada reabsorción radicular externa o interna o infección que afecte la cripta del permanente ${ }^{14}$. Es importante destacar que en los niños, por la inmadurez del sistema inmunológico, siempre está latente que la persistencia de procesos inflamatorios bacterianos, puedan producir un compromiso sistémico ${ }^{15}$.

En este estudio todas las pastas evaluadas demostraron ser efectivas, permaneciendo el $91 \%$, de las piezas tratadas libres de sintomatología clínica y con disminución radiográfica de proceso infeccioso, variando de acuerdo a la pasta utilizada. Si bien, los fracasos de las pulpotomias, en dientes primarios, rara vez producen signos y síntomas clínicos de inflamación, la principal causa de extracción, en este estudio, se debió a la presencia de movilidad excesiva asociada a reabsorción radicular externa. En el presente estudio las piezas más frecuentemente extraídas fueron los primeros molares (I superior y 3 inferiores) seguidas por los segundos molares (I superior). Aminabadin y Zoremchhingi ${ }^{2,16}$ encuentran que los primeros molares primarios presentan una anatomía compleja y variada que dificulta la accesibilidad a los conductos para la correcta instrumentación a diferencia de los segundos molares cuya accesibilidad es más sencilla no comprometiendo los tratamientos endodónticos. Tannure y colab. establecen que el principal factor responsable de los fracasos es la limitación en la técnica impuesta por las irregularidades morfológicas producidas por reabsorción inflamatoria externa debido a la persistencia de bacterias y tejido necrótico como consecuencia de procesos de desinfección incompletos ${ }^{17}$.

El oxido de zinc fue utilizado por ser una pasta ampliamente utilizada a pesar de su limitada acción antibacteriana y bajo nivel de reabsorción, permaneciendo restos del material aún después de realizado el recambio de la pieza tratada ${ }^{18}$. El éxito clínico obtenido con la pasta de Oxido de Zinc eugenol fue del $92 \%$ a los 6 meses disminuyendo al $83 \%$ a los 12 meses, lo que difiere con lo 
observado por Mani y Trairatvorakul cuyos éxito clínicos y radiográficos están entre el 48 y $53 \%$ a los 6 meses, siendo similares a lo observado a los 12 meses $^{19,20}$. Coll y Sadrian reportaron que el porcentaje de fracasos aumenta cuando el material excede el ápice ${ }^{21}$.

Las pastas yodoformadas, combinadas con diversos vehículos, han demostrado ser muy efectivas. El yodoformo es un potente agente bactericida, actuando sobre un amplio rango de bacterias, no es irritante, radiopaco y no sufre contracción. Posee acción antiséptica prolongada, utilizándose en piezas severamente infectadas, previniendo la reinfección de los conductos, por microorganismos remanentes, después de la preparación biomecánica. Es fácilmente reabsorbible no causando reacción de cuerpo extraño, permaneciendo activo químicamente hasta su completa remoción. En este estudio estas pastas demostraron éxitos entre 86 a $93 \%$, a los 12 meses, observándose un $100 \%$ de éxito con el Ultrapex, coincidiendo con lo observado por Mortazavi ${ }^{22} y$ Nurko ${ }^{23}$. Estos resultados podrían estar relacionado con la facilidad de aplicación de este material además de sus propiedades antibacterianas y la facilidad de reabsorberse rápidamente del tejido periapical. Histológicamente se demostró que la extrusión de la pasta produce una calcificación heterotópica y/o osificación en las áreas en las que penetra, por la formación de tejido osteoide que lleva al cierre del foramen apical indicando una cicatrización exitosa ${ }^{22}$. Al igual que Nurko y colab. ${ }^{24}$ se observo una reabsorción temprana de la pasta dentro del conducto sin evidencia de sintomatología clínica o radiográfica de la pieza tratada.

La pasta de Guedes Pinto, ampliamente utilizada en Brasil, en las pulpectomias en dientes primarios, contiene partes iguales de rifocort, yodoformo y paramonoclorfenol alcanforado afirmándose que posee óptimas propiedades antisépticas ${ }^{25}$. Radiográficamente se observó, en las primeras semanas, una disminución marcada de la reabsorción ósea, concordando con lo reportado por Guedes Pinto ${ }^{26}$. Nuestros resultados coinciden con lo observado con Puppin y colab quienes reportan una disminución de éxitos entre los 6 y los 12 meses, en piezas tratadas con ausencia de fistula ${ }^{27}$.

Si bien, al presente ninguna pasta ha demostrado reunir las propiedades ideales propuestas, dis- ponemos de pastas que permiten mantener las piezas dentarias primarias con pulpitis irreversibles en condiciones ópticas hasta su normal recambio siendo importante, el control clínico y radiográfico periódico a fin de evaluar la evolución del tratamiento e interceptar la aparición de procesos patológicos asociados al fracaso que pudiera afectar el desarrollo o erupción del diente permanente.

\section{Conclusiones}

Todas las pastas utilizadas demostraron ser efectivas para tratar las pulpitis irreversibles en dientes primarios no demostrandose diferencias estadísticamente significativas clínicas a los 6 y 12 meses $(p<0.2069$ vs.0.1 I 10) ni radiográficas $(p<0.3$ II 3 vs. 0.3620$)$.

La pasta de oxido de zinc eugenol demostro un éxito clínico y radiográfico a los 12 meses de $83 \%$, seguido por la Pasta de Guedes con $86 \%$, yodoformo y glicerina con $93 \%$ y el Vitapex con $100 \%$.

\section{Referencias Bibliográficas}

I. Camp J. Pediatric endodontic: Endodontic treatment for the primary and young permanent dentition. In Cohen S, Burns RC, eds. Pathways of the Pulp. 8th ed. Sr. Louis, Mo: Mosby Year Book, Inc; 20024).

2. Aminabadi NA, Farahani RM, Gajan EB. Study of root canal accessibility in human primary molars J Oral Sci. 2008; 50: 69 - 74.

3. Ringelstein D., Seow WK: The prevalence of furcation foramina in primary molars. Pediatr Dent 1989; II: 198 - 201

4. Paras LG, Rapp R, Piesco NP, Zeichner SJ, Zullo TG. An investigation of accessory canals in furcation areas of human primary molars. Part I. SEM observation of frequency, size and location of accessory foraminas in the internal and external furcation areas. J Clin Pediatr Dent 1993; 17 (2): $65-9$

5. Weisshaar S, Endodoncia en denticiones primaria y mixta. Fundamentos, patologías y diagnóstico. Quintessence (ed. esp 2003; 16 (7): 450 - 458).

6. Silva LA, Nelson-Filho P, Farias G, de SouzaGugelmin MC, Ito IY: Bacterial profile in primary teeth with necrotic pulp and periapical lesions. Braz Dent J 2006; 17: 144 - 48.

7. Coser RM, Oliveira Gondim J, Aparecida Giro EM. Evaluation of 2 endodontic techniques used 
to treat human primary molars with furcation radiolucency area: a 48 months radiographic study. Quintess Int 2008; 39: 549 - 57.

8. Holan G., Fuks AB: A comparison of pulpectomies using $\mathrm{ZOE}$ and $\mathrm{KRI}$ paste in primary molars: a retrospective study. Pediatric Dentistry 15; 403 - 407, 1993.

9. Rifkin A. A simple, effective, safe technique for the root canal treatment of abscessed primary teeth. ASDC J Dent Child 47: 435 - 4I, 1980.

10. O'Riordan MW., Coll J.: Pulpectomy procedure for deciduous teeth with severe pulpal necrosis. J Am Dent Assoc 1979; 99: 480 - 482

I I. Wrbas KT, Kielbassa AM, Hellwig E. Microscopic studies of accessory canals in primary molar furcation J Dent Child 1997; 64: I 18 - 22.

12. Vivacqua-Goms N, Gurgel-Filho ED, Gomes Bp, Ferraz CC, Zaia AA, Souza-Filho FJ. Recovery of Enterococcus faecalis after single - or multiple visit root canal treatments carried out in infected teeth ex vivo. Int Endod J 2005; 38: 697 - 704).

13. Fuks $A B$. Pulp therapy for primary and young dentitions. Dent Clin North Am 2000; 44 (3): 57I - 96.

14. American Academy of Pediatric Dentistry. Guideline on pulp therapy for primary and inmature permanent teeth. Reference Manual 2009I0. Pediatr Dent 2009; 30 (6): I79 - 86.

15. Bolan. M, Rocha MJ. Histopathologic study of physiologic pathological resoprtions in human primary teeth. Oral Surg Oral Med Oral Pathol Oral Radiol Endod 2007; 104 (5): 680 - 5.

16. Zoremchhingi, Joseph T, Varma B, Mungara.J. A Study of root canal morphology of human primary molar using computerized tomography: an in vitro study. J Indian Soc Pedod Prev Dent 2005; 23 (I): 7 - 12.

17. Tannure PN, Barcelos R, Portela MB, Gleiser R, Primo LG.Oral Surg Oral Med Oral Pathol Oral Radiol Endod. 2009 Jul; 108 (I): e29 - 33.

18. Coll JA, Sandrian R. Predicting pulpectomy success and its relationship to exfoliation and succedaneous dentition. Pediatr Dent 1996; 18: 57 - 63.
19. Mani SA, Chawla HS, Tewari A, Goyal A. Evaluation of calcium hydroxide and zinc oxide eugenol as root canal filling material in primary teeth. J Dent Child 2000; 67: 142 - 7.

20. Trairatvorakul C, Chunlasikaiwan S. Success of pulpectomies with Zinc Oxide Eugenol versus calcium hidroxide/lodofom paste in primary molars: A clinical study. 2008; 30: 303 - 308.

21. Coll JA, Sadrian R. A long-term follow on the retention rate of zinc oxide eugenol filler after primary tooth pulpectomy. Pediatr Dent 1993; 15: 249 - 53) exfoliation and succedaneous dentition. Pediatr Dent 1996; 18 (I): 57 - 63.

22. Mortazavi M, Mesbahi M. Comparison of zinc oxide and eugenol and Vitapex for root canal treatment of necrotic primary teeth. Int J Paediatr Dent 2004; 14: 417 - 24.

23. Nurko C, Garcia Godoy F: Evaluation of a calcium hydroxide/iodoforme paste (Vitapex) in root canal therapy for primary teeth J Clin Pediatr Dent 1999; 23: 289 - 94.

24. Nurko C, Ranly DM, Garcia-Godoy F, Lakshmyya KN. Resorption of a calcium hydroxide/iodoform paste (Vitapex). In root canal therapy form primary teeth: a case report Pediat Dent 2000; 22: $517-20$.

25. Ferraz Cerqueira D, Volpi Mello-Moura AC, Marcilio Santos E, Guedes Pintos AC. Cytotoxicity, Histopathological, Microbiological and clinical aspects of an endodontic iodoformbased pastes used in pediatric dentistry: a review. J Clin Pediatr Dent 2007; 32 (2): 105 - I I0.

26. Guedes Pinto AC, Paiva JG, Bozzola JR. Tratamento endodôntico de dentes decíduos com polpa mortificada. Rev Assoc Paul Cir Dent 1981; 35: 240 - 45.

27. Puppin-Rontani RMP, Peters CF, Workiczeck AT. Tratamento endodôntico de dentes deciduos com necrose pulpar. Rev Assoc Paul Cir Dent 1994; 48: 1235 - 38. 